Please do not remove this page

RMIT

UNIVERSITY

\title{
Fourier transforms from a generalised trace map
}

Horadam, Kathryn; Rao, Asha

https://researchrepository.rmit.edu.au/esploro/outputs/9921859591701341/filesAndLinks?institution=61RMIT_INST\&index=null

Horadam, K., \& Rao, A. (2006). Fourier transforms from a generalised trace map. Proceedings of the 2006 IEEE International Symposium on Information Theory, 1080-1084.

https://doi.org/10.1109/ISIT.2006.261950

Published Version: https://doi.org/10.1109/ISIT.2006.261950

Repository homepage: https://researchrepository.rmit.edu.au

(c) 2006 IEEE. Personal use of this material is permitted. However, permission to reprint/republish this material for advertising or promotional purposes or for creating new collective works for resale or redistribution to servers or lists, or to reuse any copyrighted component of this work in other works must be obtained from the IEEE.

Downloaded On 2023/04/26 21:43:17 +1000 


\title{
Fourier Transforms from a Weighted Trace Map
}

\author{
K. J. Horadam and A. Rao \\ Mathematics, SMGS \\ RMIT University \\ Melbourne VIC 3000, Australia \\ Email: kathy.horadam@ems.rmit.edu.au, asha@rmit.edu.au
}

\begin{abstract}
The class of Generalised Hadamard Transforms includes the Fourier, Generalised, Discrete Fourier, WalshHadamard, Complex Hadamard and Reverse Jacket Transforms. The Generalised Hadamard Transforms may by partly classified by signal length, by group of entries in the transform matrix and by a recently introduced third parameter, the jacket width of the transform matrix. Here we introduce a weighted trace map, which realises the Fourier Transform as an exponential weighted sum of Galois Ring traces. We give examples of Fourier Transforms with jacket width 0 , jacket width 1 and maximum jacket width (half the signal length). We show the Fourier Transforms of length $4^{k}$ with entries in $\{ \pm 1, \pm i\}$ obtained using the weighted trace map from the Galois ring $G R(4, k)$ have jacket width $2^{k-1}$.
\end{abstract}

\section{INTRODUCTION}

There are many discrete signal transforms whose transform matrices have entries on the unit circle. For instance, the Discrete Fourier Transform (DFT) and the Walsh-Hadamard Transform (WHT) are both special cases of the Fourier Transform (FT) found by interpreting the Cooley-Tukey Fast Fourier Transform in terms of abelian group characters [12]. The family of discrete Generalised Transforms $\left\{(G T)_{r}, 0 \leq r \leq\right.$ $a-1\}$ for signals of length $2^{a}[3,10.2]$ includes the WHT as case $r=0$, the complex BIFORE transform as case $r=1$ and the DFT of order $2^{a}$ as case $r=a-1$. Both the WHT and DFT are suboptimal discrete orthogonal transforms, but each has wide application for signal and image representation and processing, and efficient, easily implemented fast algorithms exist to compute them. The WHT is well-known for its applications to image compression, Walsh representation of data sequences in image coding, and for signal separation in CDMA mobile communication [7].

The transform matrices of all these transforms fall into the class of Butson Hadamard (BH) matrices [2].

For multi-phase or multilevel signals where entries outside the complex $n^{t h}$ roots of unity are needed, the first author generalised the class of $\mathrm{BH}$ matrices to define a Generalised Hadamard Transform (GHT) [6]. The GHT includes all the above transforms and other families, such as the unimodular complex Hadamard transforms, whose matrices have entries on the complex unit circle, and the Reverse Jacket Transforms of [10]. The former are important in high-energy and theoretical physics and play a critical role in the theory of quantum information [17]. The latter are so-called because the unitary matrix representing the transform has a border ("jacket") and centre which switch some elements under inversion. This family includes the weighted Hadamard Transforms [11], with a border of $\{ \pm 1\}$ and real centre entries of absolute value greater than 1 , which weight the mid-band frequencies of the signal more.

In [6], those GHT matrices which had a border of $\{ \pm 1\}$ were called jacket matrices. The maximum width of a border of $\{ \pm 1\}$ in a generalised $\mathrm{BH}$ matrix is proposed here as a new parameter for classifying transforms. In particular it can be applied to Fourier Transforms, and ranges from 0 or 1 for DFTs, depending whether the signal length is odd or even, to the maximum possible for WHTs.

In Section II, a description of GHT matrices and their properties is summarised from [6]. Each normalised GHT matrix has an associated jacket width $w \geq 0$. (We have modified the definition of [6] slightly.) This is the maximum width of a complete border of rows and columns consisting entirely of \pm 1 s in the normalised GHT matrix, up to permutations of the rows and columns.

In Section III we restrict to GHT with entries which are roots-of-unity, so the GHT matrix is a $\mathrm{BH}$ matrix. We introduce a weighted trace map which represents the Fourier Transform (FT) as an exponential weighted sum of Galois Ring trace maps. Examples of jacket transforms of varying widths and signal alphabets are derived. In Section IV we summarise our results and pose further problems.

\section{Generalised HADAMARD TRANSFORMS}

In this most general situation, we work with a signal alphabet from a ring $R$ with unity 1 , and a transform matrix with entries from its group of units $R^{*}$. This includes signal alphabets from the real field $\mathbb{R}$, the complex field $\mathbb{C}$ and the Galois Fields $G F\left(p^{e}\right)$. It also includes Galois Ring alphabets $G R\left(p^{e}, k\right)$, in which case the group of units is $\left\{\sum_{j=0}^{e-1} p^{j} u_{j}: u_{j} \in \mathcal{T}, u_{0} \neq 0\right\}$, where $\mathcal{T}$ is the Teichmüller set [13]. If, however, we need to distinguish signal values $x$ and $-x$, the ring $R$ must have characteristic $\neq 2$. The transform matrix is a Generalised Butson Hadamard (GBH) matrix.

Definition 2.1: Suppose $R$ is a ring with unity 1 , group of units $R^{*}$ and that char $R$ does not divide $v$. A square matrix $M$ of order $v \geq 2$, with entries from a subgroup $N \leq R^{*}$, is a Generalised Butson Hadamard (GBH) matrix, if

$$
M M^{*}=M^{*} M=v I_{v},
$$


where $M^{*}$ is the transpose of the matrix of inverse elements of $M: \quad m_{i j}^{*}=\left(m_{j i}\right)^{-1}$. It is denoted $\operatorname{GBH}(N, v)$, or $G B H(|N|, v)$ if $N$ is finite.

If $\mathbf{x}$ is a signal of length $v$ from $R$, where $v \in R^{*}$, and $M$ is a $\operatorname{GBH}(N, v)$, a Generalised Hadamard Transform (GHT) of $\mathrm{x}$ is

$$
\hat{\mathbf{x}}=M \mathbf{x}
$$

and an Inverse Generalised Hadamard Transform (IGHT) of $\hat{\mathbf{x}}$ is

$$
\mathbf{x}=v^{-1} M^{*} \hat{\mathbf{x}}
$$

A $G B H$ matrix is always equivalent to a normalised $G B H$ matrix, which has first row and column consisting of all 1s. By taking the inner product of any non-initial row of a normalised $G B H$ matrix $M$ with the all-1s first column of $M^{*}$, we see that the sum of the entries in any row of $M$, apart from the first, must equal 0 , and similarly for rows of $M^{*}$ (columns of the matrix of inverses $M^{(-1)}=\left[m_{i j}^{-1}\right]$ ). The tensor product of two $G B H$ matrices over the same group $N$ is a $G B H$ matrix over $N$.

Throughout this section, let $C$ be an indexing set of order $v$ (sometimes $C$ is an abelian group such as the group $\mathbb{Z}_{v}$ of integers $\bmod v$ or the direct product $\mathbb{Z}_{2}^{e}$ of $e$ copies of $\mathbb{Z}_{2}$, but $C$ may be non-abelian or the group structure may be irrelevant). Let $D_{n} \leq \mathbb{C}^{*}$ denote the group of complex $n^{t h}$ roots of unity.

Definition 2.2: Let $R$ be a ring with unity 1. A normalised $G B H(N, v)$ matrix $K$ indexed by $C=\{1, \ldots, v\}$ with entries from $N \leq R^{*}$ is a jacket matrix if it is permutation equivalent to a matrix of the form

$$
\widetilde{K}=\left[\begin{array}{rrrrr}
1 & 1 & \ldots & 1 & 1 \\
1 & * & \ldots & * & \pm 1 \\
\vdots & \vdots & \vdots & \vdots & \vdots \\
1 & * & \ldots & * & \pm 1 \\
1 & \pm 1 & \ldots & \pm 1 & \pm 1
\end{array}\right]
$$

where the central entries $*$ are from $N$. The jacket width of $K$ is $w \geq 1$ if $K$ is permutation equivalent to a jacket matrix $\widetilde{K}$ in which rows $1, \ldots, w, v-w+1, \ldots, v$ and columns $1, \ldots, w, v-w+1, \ldots, v$ all consist of \pm 1 and $w$ is maximal for this property. If $K$ is not permutation equivalent to any jacket matrix it has jacket width 0 .

Since all non-initial \pm 1 rows and columns sum to 0 in $R$, the order $v$ of a jacket matrix $K$ must be even.

Corollary 2.3: If $v$ is odd, any normalised $\operatorname{GBH}(N, v)$ matrix has jacket width 0 .

The family of Discrete Fourier Transform (DFT) matrices of order $v$

$$
\mathcal{F}_{v}=\left[\omega^{j k}\right]_{0 \leq j, k \leq v-1}
$$

where $\omega=e^{-2 \pi i / v}$, provides examples of jacket width 1 in every even order.

Example 2.4: The DFT matrix $\mathcal{F}_{2 n}$ of order $2 n$ is permutation equivalent to a jacket matrix of width 1 with entries in $D_{2 n}$.

Proof. By [10, Theorem 1, Definition 1] $\mathcal{F}_{2 n}$ is permutation equivalent to a jacket matrix of width $\geq 1$. But for a fixed $j$, $\omega^{j k}= \pm 1$ for all $0 \leq k \leq 2 n-1$ if and only if $\omega^{j}= \pm 1$ if and only if either $j=0$ (the initial row and column) or $j=n$. Thus the width is exactly 1 .

At the other extreme, the WHT matrix $\mathcal{S}_{t}$ of order $2^{t}$

$$
\mathcal{S}_{1}=\left[\begin{array}{rr}
1 & 1 \\
1 & -1
\end{array}\right], \quad \mathcal{S}_{t}=\bigotimes^{t} \mathcal{S}_{1}, t \geq 2,
$$

with jacket width $2^{t-1}$, is "all jacket". In fact this holds for any Hadamard matrix transform.

Example 2.5: A jacket matrix $K$ of order $2 n$ has maximum width $n$ if and only $K$ is a normalised Hadamard matrix.

This means a maximal width jacket matrix of order $2 n$ has entries in $D_{2}=\{ \pm 1\}$ and either it is $\mathcal{S}_{1}$ or $n$ is even.

In [6, Lemma 3.5] it is shown that if $K_{i}$ is a width $w_{i} \geq 1$ jacket matrix with entries from $R^{*}$, for $i=1,2$, then their tensor product $K_{1} \otimes K_{2}$ is a jacket matrix of width at least $2 w_{1} w_{2}$. A similar argument shows that if $w_{i}=0$ then $K_{1} \otimes K_{2}$ has width at least $w_{j}(j \neq i)$.

Theorem 2.6: If $K_{i}$ is a normalised $\operatorname{GBH}\left(N, v_{i}\right)$ matrix of jacket width $w_{i}$ with entries from $R^{*}$, for $i=1,2$, then $K_{1} \otimes K_{2}$ is a jacket matrix of width at least $2 w_{1} w_{2}$, if $w_{1} w_{2} \geq 1$, and has jacket width at least $w_{j}(j \neq i)$, if $w_{i}=0$.

Consequently we are interested in finding the fundamental jacket matrices, which are not permutation equivalent to any such tensor products.

Definition 2.7: [6, Definition 4.1] A jacket matrix of order $2 n$ is a primary jacket matrix $K_{n}$ if it is minimal with respect to tensor product, that is, there are no jacket matrices $K, K^{\prime}$ such that $K_{n}$ is permutation equivalent to $K \otimes K^{\prime}$.

Clearly any jacket matrix of width 1 , such as the even length DFTs of Example 2.4, is primary, but by Example 2.5 this sufficient condition is not necessary for a jacket matrix to be primary.

Corollary 2.8: A jacket matrix of width 1 is primary. A jacket matrix of order $2 n \geq 4$ and maximum width $n=2 k$ is primary whenever $k$ is odd.

The tensor product of two jacket matrices is a jacket matrix, but by Theorem 2.6, for a tensor product of normalised $G B H$ 
matrices to be jacket matrix it is enough that one factor is a jacket matrix.

Corollary 2.9: [6, Theorem 5.1] Let $B$ be a normalised $G B H$ matrix and $K$ a jacket matrix, both with entries in $R^{*}$. Then $B \otimes K$ is a jacket matrix.

The width $w$ of a GHT matrix is a third parameter, after $v$ and $N$, we can use to construct and classify signal transforms over varying signal alphabets.

\section{CONSTRUCTION OF ROOT-OF-UNITY TRANSFORMS AND THE WEIGHTED TRACE MAP}

In this section we concentrate on transforms whose entries are all complex $m^{t h}$ roots of unity, so $N=D_{m}$. The transform matrices are all $\mathrm{BH}$ matrices $\operatorname{GBH}(m, v)$. From now on, let the index set $C$ be an abelian group of order $v$ and exponent $m$. (The exponent $m \geq 0$ of a group $G$ which is written multiplicatively with identity 1 , is the smallest integer such that $g^{m}=1$ for every $g \in G$, so, for example, $\mathbb{Z}_{v}$ has exponent $v$ and $\mathbb{Z}_{2}^{e}$ has exponent 2).

The $B H$ matrix (4) of the DFT for signals of length $v$ uses as indexing set the cyclic group $C=\mathbb{Z}_{v}$ of exponent $v$. The construction generalises for any finite abelian group $C$ of order $v$ to a Fourier Transform (FT) matrix [12].

An irreducible character of a finite abelian group $C$ of order $v$ and exponent $m$ is any group homomorphism from $C$ to the multiplicative group $D_{m}=\left\langle e^{2 i \pi / m}\right\rangle \subset \mathbb{C}$ of all complex $m^{\text {th }}$ roots of unity. The character group $\widehat{C}=\operatorname{Hom}\left(C, D_{m}\right)$ of all irreducible characters of $C$ is isomorphic to $C$.

Definition 3.1: Let $C$ be a finite abelian group of order $v$ and exponent $m$ and fix an ordering $C=\left\{c_{1}, \ldots, c_{v}\right\}$. Fix an isomorphism $\chi: C \rightarrow \widehat{C}$ and denote the image of $c \in C$ by $\chi_{c}$. The Fourier Transform (FT) of a complex-valued function $\varphi: C \rightarrow \mathbb{C}$ is the function $\widehat{\varphi}: C \rightarrow \mathbb{C}$ given by

$$
\widehat{\varphi}\left(c_{k}\right)=\sum_{\ell=1}^{v} \varphi\left(c_{\ell}\right) \chi_{c_{k}}\left(c_{\ell}\right), 1 \leq k \leq v
$$

and the Inverse Fourier Transform (IFT) of $\widehat{\varphi}$ is

$$
\varphi\left(c_{\ell}\right)=v^{-1} \sum_{k=1}^{v} \widehat{\varphi}\left(c_{k}\right) \overline{\chi_{c_{k}}\left(c_{\ell}\right)}, 1 \leq \ell \leq v,
$$

where $\bar{z}$ denotes the complex conjugate of $z$. The FT matrix, with entries in $D_{m}$,

$$
\mathcal{F}_{C}=\left[\chi_{c_{k}}\left(c_{\ell}\right)\right]_{1 \leq k, \ell \leq v}
$$

is a $\operatorname{GBH}\left(D_{m}, v\right)$.

When $C=\mathbb{Z}_{v}$, the FT is the usual DFT of (4). When $C=\mathbb{Z}_{2}^{a}$, the FT is the WHT of (5), since any homomorphism is represented by a linear Boolean function $L_{\mathbf{u}}: \mathbb{Z}_{2}^{a} \rightarrow \mathbb{Z}_{2}$ with $L_{\mathbf{u}}(\mathbf{v})=\langle\mathbf{u}, \mathbf{v}\rangle$, where $\langle\mathbf{u}, \mathbf{v}\rangle$ is the inner product of vectors $\mathbf{u}$ and $\mathbf{v}$ over $G F(2)$, and vice versa.

Any two abelian groups $C, C^{\prime}$ of the same order $v$ but different exponents $m, m^{\prime}$, respectively, will determine Fourier Transforms with entries in distinct roots-of-unity subgroups $D_{m}, D_{m^{\prime}}$, respectively. For each $v$, there are at most finitely many such transforms. The simplest illustration is given for $v=4$ by $C=\mathbb{Z}_{4}$ of exponent 4 (the DFT) and $C^{\prime}=\mathbb{Z}_{2}^{2}$ of exponent 2 (the WHT).

More subtly, any two non-isomorphic abelian groups of the same order $v$ and exponent $m$, will determine different Fourier Transforms. Again, there are only finitely many such transforms. A small example for $v=16$ and $m=4$ is given by $C=\mathbb{Z}_{4} \times \mathbb{Z}_{4}$ and $C^{\prime}=\mathbb{Z}_{4} \times \mathbb{Z}_{2}^{2}$.

Thirdly, an abelian group $C$ of order $v$ and exponent $m$ may have several distinct formulations as a direct product. This is the basis of the Cooley-Tukey FFT and is exemplified by $C=\mathbb{Z}_{6} \cong \mathbb{Z}_{2} \times \mathbb{Z}_{3}$.

Finally, for each $C$, the precise form of the FT will depend on the choice of isomorphism $\chi: C \rightarrow \widehat{C}$ in (6).

In the general case, we write $C$ as a direct product. If $C \nsubseteq \mathbb{Z}_{p^{e}}$ for any prime power $p^{e}$, then $C$ has at least one decomposition as a direct sum of proper subgroups. Write $C=C_{1} \oplus \cdots \oplus C_{n} \cong C_{1} \times \cdots \times C_{n}$, using the standard isomorphism between a finite internal direct sum and external direct product. There may be several ways of doing this.

For example, by the fundamental theorem for finite abelian groups, we may assume, if required, that $C_{j}=\mathbb{Z}_{m_{j}}$ is cyclic and $m_{j} \mid m$, where $m$ is the exponent of $C$. In this case, the isomorphism $\chi: C \rightarrow \widehat{C}$ may be selected as follows [7]. Write $x_{j}=(0, \ldots, 0,1,0, \ldots, 0)$ for the standard generator of the $j^{t h}$ direct summand of $C$ and select $\omega_{j}=\omega^{m / m_{j}}=e^{2 i \pi / m_{j}}$ as the $m_{j}^{\text {th }}$ root of unity used to define the character group $\widehat{C_{j}}$. Then, for all $\mathbf{c}=\left(c_{1}, c_{2}, \ldots, c_{n}\right), \mathbf{b}=\left(b_{1}, b_{2}, \ldots, b_{n}\right) \in C$,

$$
\begin{aligned}
\chi_{\mathbf{c}}(\mathbf{b}) & =\chi_{\mathbf{c}}\left(\sum_{j=1}^{n} b_{j} x_{j}\right)=\prod_{j=1}^{n}\left(\chi_{\mathbf{c}} \downarrow C_{j}\right)\left(b_{j} x_{j}\right) \\
& =\prod_{j=1}^{n} \chi_{\mathbf{c}_{\mathbf{j}}}\left(b_{j}\right)=\prod_{j=1}^{n} \omega_{j}^{c_{j} b_{j}}
\end{aligned}
$$

by $\left[9\right.$, Chapter 9], where the character $\left(\chi_{\mathbf{c}} \downarrow C_{j}\right)$ of the subgroup $C_{j}$ is the restriction of $\chi_{\mathbf{c}}$ to $C_{j}$. That is, for all $\mathbf{c}=\left(c_{1}, c_{2}, \ldots, c_{n}\right), \mathbf{b}=\left(b_{1}, b_{2}, \ldots, b_{n}\right) \in C$,

$$
\chi_{\mathbf{c}}(\mathbf{b})=\omega^{\mathbf{c} * \mathbf{b}}, \text { where } \mathbf{c} * \mathbf{b}=\sum_{j=1}^{n} c_{j} b_{j} m / m_{j} .
$$

In particular, when $m_{j}=m$ for all $1 \leq j \leq n$, $\mathbf{c} * \mathbf{b}=\mathbf{c} \cdot \mathbf{b}=\sum_{j=1}^{n} c_{j} b_{j}$.

Now we present a new formulation of the isomorphism $\chi$ : $C \rightarrow \widehat{C}$ in terms of Galois Ring traces. We no longer require all the $C_{j}$ to be cyclic. Instead, assume that, for each $j, C_{j}=$ 
$\left(\mathbb{Z}_{m_{j}}\right)^{k_{j}}$, where $m_{j}=p_{j}^{e_{j}}$ is a prime power. The primes $p_{j}$ are not necessarily distinct. In this case, each character can be realised in terms of trace maps for Galois Rings. The Galois Ring $G R\left(p^{e}, k\right)$ is a degree $k$ Galois extension of the ring $\mathbb{Z}_{p^{e}}$. See McDonald [13] for example, for more details on Galois Rings, or Hammons et al [4] for a brief outline in the case $p^{e}=4$.

The trace map for the Galois $\operatorname{Ring} G R\left(p^{e}, k\right)$ is defined as follows. Using the $p$-adic representation $u=\sum_{j=0}^{e-1} p^{j} u_{j}$ of elements of $G R\left(p^{e}, k\right)$, the Frobenius automorphism $f$ : $G R\left(p^{e}, k\right) \rightarrow G R\left(p^{e}, k\right)$ is

$$
f(u)=\sum_{i=0}^{e-1} p^{i} u_{i}^{p} .
$$

When $e=1, G R(p, k)$ is the Galois Field $G F\left(p^{k}\right)$ and $f$ is the usual Frobenius automorphism for $G F\left(p^{k}\right)$. The trace $\operatorname{map} T_{\left(p^{e}, k\right)}: G R\left(p^{e}, k\right) \rightarrow \mathbb{Z}_{p^{e}}$ over $G R\left(p^{e}, k\right)$ is defined by

$$
T_{\left(p^{e}, k\right)}(u)=u+f(u)+f^{2}(u)+\ldots+f^{k-1}(u) .
$$

It is easy to see that the trace map satisfies the following properties: for any $u, v \in G R\left(p^{e}, k\right)$ and $\alpha \in \mathbb{Z}_{p^{e}}$,

1) $T_{\left(p^{e}, k\right)}(u+v)=T_{\left(p^{e}, k\right)}(u)+T_{\left(p^{e}, k\right)}(v)$.

2) $T_{\left(p^{e}, k\right)}(\alpha u)=\alpha T_{\left(p^{e}, k\right)}(u)$.

3) $T_{\left(p^{e}, k\right)}$ is surjective.

As well, $T_{\left(p^{e}, k\right)}$ is equi-distributed. Note that $u \in G R\left(p^{e}, k\right)$ is invertible if and only if $u_{0} \neq 0$. Thus every noninvertible element of $G R\left(p^{e}, k\right)$ can be written uniquely as $u=\sum_{i=j}^{e-1} p^{i} u_{i}$ for a smallest $j=1,2, \ldots, e-1$ for which $u_{j} \neq 0$, so we can represent elements of $G R\left(p^{e}, k\right)$ by $u^{(j)}=\sum_{i=j}^{e-1} p^{i} u_{i}, \quad j=0,1,2, \ldots, e-1$.

Theorem 3.2: [16, Lemma 2.1] Let $B_{j}=\left\{p^{j} t \mid t=\right.$ $\left.0,1,2, \ldots, p^{e-j}-1\right\}, j=0,1,2, \ldots, e-1$. For each $j=0,1,2, \ldots, e-1$, as $x$ ranges over $G R\left(p^{e}, k\right)$, $T_{\left(p^{e}, k\right)}\left(x u^{(j)}\right)$ takes elements in $B_{j}$ equally often; that is, $p^{e(k-1)+j}$ times.

Importantly, since the additive group of $G R\left(p^{e}, k\right)$ is isomorphic to $\left(\mathbb{Z}_{p^{e}}\right)^{k}$, of order $p^{e k}$ and exponent $p^{e}$, we know by 1 ) above that each additive character of $\left(\mathbb{Z}_{p^{e}}\right)^{k}$ may be realised in terms of the corresponding trace map:

$$
\chi_{u}(v)=\omega^{T_{\left(p^{e}, k\right)}(u v)}, \quad u, v \in G R\left(p^{e}, k\right), \quad \omega=e^{2 \pi i / p^{e}} .
$$

Now we can use (7) and (9) to realise each character of $C$ as a weighted trace map, written as a weighted sum of Galois Ring traces. This map first appears in [14], for the case $k_{1}=k_{2}=\cdots=k_{n}$, where it is called the trace-like map.

Definition 3.3: For $1 \leq j \leq n$ let $p_{j}$ be prime such that $p_{1} \leq p_{2} \leq \cdots \leq p_{n}$. Let $m_{j}=p_{j}^{e_{j}}$ and let $C_{j}=\left(\mathbb{Z}_{m_{j}}\right)^{k_{j}}$. For each $1 \leq j \leq n$, let $T_{\left(m_{j}, k_{j}\right)}: G R\left(m_{j}, k_{j}\right) \cong C_{j} \rightarrow \mathbb{Z}_{m_{j}}$ be the corresponding trace map of (8).
Let $C=C_{1} \times \cdots \times C_{n}$ have exponent $m$ and let $\omega=$ $e^{2 i \pi / m}$. Define the weighted trace map $T: C \rightarrow \mathbb{Z}_{m}$ by

$$
T(\mathbf{b})=\sum_{j=1}^{n} T_{\left(m_{j}, k_{j}\right)}\left(b_{j}\right) m / m_{j}, \mathbf{b} \in C .
$$

Therefore, for each fixed $\mathbf{c} \in C$,

$$
T(\mathbf{c b})=\sum_{j=1}^{n} T_{\left(m_{j}, k_{j}\right)}\left(c_{j} b_{j}\right) m / m_{j}, \quad \mathbf{b} \in C
$$

is a distinct homomorphism from $C$ to $\mathbb{Z}_{m}$.

Theorem 3.4: Let $C$ and $T$ be as in Definition 3.3. For each c $\in C$, the isomorphism $\chi: C \rightarrow \widehat{C}$ may be selected as

$$
\chi_{\mathbf{c}}(\mathbf{b})=\omega^{T(\mathbf{c b})}, \quad \mathbf{b} \in C .
$$

The corresponding FT matrix, with entries in $D_{m}$, is

$$
\mathcal{F}_{C}=\left[\omega^{T(\mathbf{c b})}\right]_{\mathbf{c}, \mathbf{b} \in C}
$$

These Fourier Transform matrices (13) are all instances of a class of matrices called cocyclic, investigated by the authors and colleagues over the past decade [5], [8], [14].

We finish by determining the jacket widths of several infinite families (indexed by signal length) of root-of-unity transforms. The first two follow immediately from Corollary 2.3 and Theorem 2.6.

Example 3.5: Let $C$ be an abelian group of odd order $v$ and exponent $m$. Then the Fourier Transform matrix $\mathcal{F}_{C}$ has jacket width 0 . The DFT of (4) is the case $C=\mathbb{Z}_{v}$.

Example 3.6: For any $\mathcal{F}_{C}$ of jacket width 0 in Example 3.5 , the tensor product $\mathcal{S}_{1} \otimes \mathcal{F}_{C}$ has jacket width 1 . The index group is $\mathbb{Z}_{2} \times C$, of order $2 v$ and exponent $2 m$. If $C=\mathbb{Z}_{v}$ then $\mathbb{Z}_{2} \times C \cong \mathbb{Z}_{2 v}$ and $\mathcal{S}_{1} \otimes \mathcal{F}_{C}$ can be permuted to the DFT matrix $\mathcal{F}_{2 v}$ of (4).

The last example gives a family of Fourier Transforms with entries in $\{ \pm 1, \pm i\}$, of orders $4^{k}, k \geq 1$ and jacket width $2^{k-1}$.

Lemma 3.7: Let $C=C_{1}=\left(\mathbb{Z}_{4}\right)^{k}$, of order $4^{k}$ and exponent 4 , so $T=T_{\left(2^{2}, k\right)}$ in Definition 3.3. Then $\mathcal{F}_{C}$ is a jacket matrix of width $2^{\grave{k-1}}$, with entries in $\{ \pm 1, \pm i\}$.

Proof. If $c$ is not invertible then $T_{\left(2^{2}, k\right)}(c b)$ takes values 0 and 2 equally often, by Theorem 3.2. Hence $\chi_{c}(b)$ will be a row consisting only of \pm 1 .

By the 2-adic representation, $c=2 t$ where $t \in \mathcal{T}$, the Teichmüller set and $|\mathcal{T}|=2^{k}$.

The jacket width and other properties of an arbitrary Fourier Transform matrix (13) is currently under investigation. 


\section{SUMMARY AND FURTHER WORK}

We have shown that after signal length $v$ and entry alphabet group $N$, a third parameter, jacket width $w \geq 0$, may be used to classify Generalised Hadamard Transforms. For real signals, the real-valued centre-weighted Hadamard Transforms, with jacket width at least 1 , weights the mid-band frequencies of the signal more than the WHT does.

For complex signals of length $v$ we have shown that for any abelian group $C$ of order $v$ and exponent $m$ there is a Fourier Transform $\left[\left(e^{2 i \pi / m}\right)^{T(\mathbf{c b})}\right] \mathbf{c}, \mathbf{b} \in C$ defined by a weighted trace map $T: C \rightarrow \mathbb{Z}_{m}$, which is a weighted sum of Galois Ring traces. Since abelian groups of order $v$ can have different exponents, or the same exponent but different decompositions as direct products, in most cases there is a choice of Fourier Transforms.

The following problems could next be considered.

What properties of a signal over complex-valued alphabets $N=D_{m}$ or $N=\mathbb{C}^{*}$, say, does the jacket width of a Generalised Hadamard Transform measure?

There are infinite families (indexed by order $2 n$ ) of primary jacket matrices with minimum jacket width $1\left(N=\mathbb{Z}_{2 n}\right)$ and with maximum jacket width $n=2 k, k$ odd $(N=\{ \pm 1\})$. Given $v=2 n$, do primary jacket matrices exist for some $N$ in all possible jacket widths $w=1,2, \ldots, n$ ?

Jacket width is defined for normalised $\operatorname{GBH}(N, v)$ up to permutation equivalence. If all equivalence operations on a $G B H(N, v)$ are allowed, i.e. left multiplication of a row or right multiplication of a column by an element of $N$, could the jacket width change? In other words, is jacket width an invariant of each equivalence class of $\operatorname{GBH}(N, v)$ ?

Fourier Transforms admit fast decompositions. For a given $v$, what is the optimal FFT obtained from the various possible FT of Theorem 3.4?

Finally, how do the GHTs above relate to the algebraic classification of Fourier-type signal transforms in [15]?

\section{REFERENCES}

[1] K. T. Arasu and W. de Launey, Two-dimensional perfect quaternary arrays, IEEE Trans. Inform. Theory 47(4) (2001) 1482-1493.

[2] A. T. Butson, Generalised Hadamard matrices, Proc. Amer. Math. Soc. 13 (1962) 894-898.

[3] D. F. Elliott and K. R. Rao, Fast Transforms: Algorithms, Analyses, Applications, Academic Press, New York, 1982.

[4] A. R. Hammons, P. V. Kumar, A. R. Calderbank, N. J. A. Sloane and P. Solé, The $\mathbb{Z}_{4}$-linearity of Kerdock, Preparata, Goethals, and related codes, IEEE Trans. Inform. Theory 40 (1994) 301-319.

[5] K. J. Horadam, An introduction to cocyclic generalised Hadamard matrices, Discrete Appl. Math. 102 (2000) 115-131.

[6] K. J. Horadam, A generalised Hadamard Transform, Proc. 2005 IEEE International Symposium on Information Theory, 4-9 September 2005, Adelaide, Australia, 1006-1008.

[7] K. J. Horadam, Hadamard Matrices and Their Applications, PUP, Princeton, 2006, to appear.

[8] K. J. Horadam and P. Udaya, Cocyclic Hadamard codes, IEEE Trans. Inform. Theory 46 (4) (2000) 1545-1550.

[9] G. James and M. Liebeck, Representations and Characters of Groups, C.U.P., Cambridge, 1993.

[10] M. H. Lee, B. Sunder Rajan and J. Y. Park, A generalized reverse jacket transform, IEEE Trans. Circuits Syst. II, 48 (7) (2001) 684-690.
[11] S.-R. Lee and M. H. Lee, On the reverse jacket matrix for weighted Hadamard Transform, IEEE Trans. Circuits Syst. II, 45 (3) (1998) 436441.

[12] D. K. Maslen and D. N. Rockmore, Generalised FFTs - a survey of some recent results, DIMACS Ser. Discr. Math. Theoret. Comp. Sci. 28 (1997) 183-237.

[13] B. R. McDonald, Finite rings with identity, Marcel Dekker, New York, 1974.

[14] N. Pinnawala and A. Rao, Cocyclic Codes over $\mathbb{Z}_{n}$, Res. Rept. 2005/03, Math. Dept., RMIT University, 2005.

[15] M. Puschel and J. M. F. Moura, The algebraic structure in signal processing: time and space, preprint 2005.

[16] A. Rao and N. Pinnawala, New linear codes over $\mathbb{Z}_{p^{s}}$ via the trace map, Proc. 2005 IEEE International Symposium on Information Theory, 4-9 September 2005, Adelaide, Australia, 124-126.

[17] W. Tadej and K. Zyczkowski, A concise guide to complex Hadamard matrices, preprint 2005, arXiv:quant-ph/0512154v1, 19 Dec 2005. 